\title{
Efficacy and safety of dissimilar acupuncture intervention time-points in treating stroke: a systematic review and network meta-analysis
}

\author{
Yue Zhuo, Ming Xu, Shifeng Deng, Yuchen Zhang, Xiaoye Lu, Boyu Wu, Hong Zhang \\ College of Acupuncture-Moxibustion and Tuina, Hunan University of Chinese Medicine, Changsha, China \\ Contributions: (I) Conception and design: Y Zhuo, H Zhang; (II) Administrative support: H Zhang; (III) Provision of study materials or \\ patients: H Zhang; (IV) Collection and assembly of data: Y Zhuo, B Wu, M Xu; (V) Data analysis and interpretation: S Deng, Y Zhuo, Y Zhang, X Lu; \\ (VI) Manuscript writing: All authors; (VII) Final approval of the manuscript: All authors. \\ Correspondence to: Hong Zhang, PhD. College of Acupuncture-Moxibustion and Tuina, Hunan University of Chinese Medicine, No.300 Xueshi \\ Road, Hanpu Science and Education Park, Yue Lu District, Changsha 410208, China. Email: zh5381271@sina.com.
}

Backgroundk Impairment of motor function and activities of daily living is very common in post-stroke
patients. Acupuncture has been used in stroke for a long time. Although growing evidence supports the
beneficial effects of acupuncture, there is still no clear conclusion on the optimal intervention time-point for
acupuncture in stroke. Thus, we tried to assess the efficacy and safety of dissimilar acupuncture intervention
time-points by network meta-analysis (NMA).

Methods: A retrieval was performed in several databases from beginning to October 2020. We only enrolled randomized controlled trials (RCTs) testing acupuncture for stroke, and the outcome indicators measured were Fugl-Meyer assessment (FMA) score, Barthel Index (BI), and adverse events.

Results: Thirty-eight trials involving 3,836 participants were included in this NMA, the vast majority of which had a low or unclear risk of selection bias, detection bias, reporting bias, attrition bias, but had a high risk of performance bias. The results showed as following: (I) acupuncture treatment (Acu) is significantly more effective than non-acupuncture treatment (Non-Acu) with a good safety. (II) In terms of FMA, acupuncture within $48 \mathrm{~h}$ post-stroke (Acu I) was superior to acupuncture within 2-15 d post-stroke (Acu II) [standard mean difference (SMD): 7.17; 95\% confidence interval (CI), 1.11 to 13.22], acupuncture within 16-30 d post-stroke (Acu III) (SMD: 20.73; 95\% CI, 13.68 to 27.78), acupuncture within 1 month to half a year post-stroke (Acu IV) (SMD: 26.95; 95\% CI, 14.88 to 39.02). As for BI, Acu I was the optimal timepoint, comparing with Acu III (SMD: 15.18; 95\% CI, 8.97 to 21.39) and Acu IV (SMD: 22.88; 95\% CI, 11.07 to 34.69 ). (III) Results of ranking indicated that Acu was better than Non-Acu at a similar stage in improving FMA and BI, while Acu I was the optimal intervention time-point, followed by Acu II.

Conclusions: Although the credibility of our conclusions is low, our NMA indicates that acupuncture can be beneficial for stroke survivors with good safety. In terms of improvement in FMA score and BI, the soon use of acupuncture the better the efficacy. The optimal acupuncture intervention time-point for stroke is within $48 \mathrm{~h}$ post-stroke, and the significant validity period lasts until 15 days after onset.

Keywords: Acupuncture; stroke; early medical intervention; systematic review; network meta-analysis (NMA)

Submitted May 04, 2021. Accepted for publication Aug 09, 2021.

doi: 10.21037/apm-21-1127

View this article at: https://dx.doi.org/10.21037/apm-21-1127 


\section{Introduction}

Stroke, an increasing public health problem worldwide, which seriously affects patients' physical and mental wellbeing as well as the quality of life (1). Previous studies have revealed that stroke is the second most common source of mortality and a leading source of disability in adults all over the world, also showing a younger trend (2). In China, due to an aging population and insufficiency of medical resources, the incidence of stroke has risen significantly and become the first cause of death (3). Neurologic sequelae, such as impairments in motor, sensory, and cognitive functions are common among post-stroke survivors, consequently, their activities of daily living are severely compromised. Therefore, stroke is not only an individual health concern, but also finally brings about an enormous burden on their families as well as the health care system (4).

The prevention and treatment of stroke have been a challenge to us for a long time. With the advancement of medical science, methods such as surgical operations, pharmaceuticals, and rehabilitation interventions have been widely used in the process of stroke according to the individual condition (5). However, these approaches also have aroused the concerns such as the invasive risk of the surgery, side effects of the drugs, increasing costs of interventions (6). Meanwhile, how to deal with post-stroke sequelae remains to be a severe problem. It is impossible to ignore the consistently high incidence of motor dysfunction and impairment in the activities of daily living after stroke (7).

Acupuncture, an important part of Chinese medicine, has been used as an alternative and complementary therapy in the clinical treatment of a wide range of diseases. Nowadays, there has been a growing concern about acupuncture for dealing with stroke (8-10), besides, some evidence indicated that acupuncture can be very helpful in the improvement of limb motor dysfunction and quality of life for post-stroke patients (11-13). The promotion of cytogenesis and neurogenesis in the lesioned area as well as the modulation of neurochemicals induced by acupuncture are considered to be its main therapeutic mechanism $(14,15)$. However, the quality of related studies is uneven $(16,17)$, apart from this, most studies have focused on comparing the efficacy of different acupuncture therapies in post-stroke patients, with few high-quality studies on the optimal time-point of acupuncture interventions. Until now, there has been no clear conclusion about when should acupuncture be adopted to maximize the benefit to patients with stroke. It is obvious that a deeper exploration of this issue will greatly facilitate the application and development of acupuncture.

In recent years, the number of randomized controlled trials (RCTs) of acupuncture therapy on stroke has been a gradual increase and the acupuncture intervention timepoint varies from the early stage of the stroke to the advanced stage, accordingly, the outcomes are different (18-20). However, we can figure out which is the best acupuncture intervention time-points for post-stroke patients by processing these clinical data through Bayesian network meta-analysis (NMA). This work will help patients recuperate better and the result is expected to save more health resources. Therefore, we designed the NMA of RCTs in the hope of providing an evidence-based reference for both clinicians and researchers.

\section{Methods}

The study was performed strictly according to the Preferred Reporting Items for Systematic Reviews and Meta-Analyses for Network Meta-Analysis (PRISMA-NMA) guidelines and its extension statement for NMA as well (21) (available at https://dx.doi.org/10.21037/apm-21-1127). We have followed the protocol that was registered in INPLASY (INPLASY2020120060), which is available from https:// inplasy.com/inplasy-2020-12-0060/.

\section{Data sources and search strategy}

A comprehensive search for potentially relevant literature has been performed in seven online databases from their establishment to October 2020: the Cochrane Library, PubMed, EMBASE Database, China Biological Medicine Database (CBM), Chinese Scientific Journals Database (VIP), Wan Fang databases and China National Knowledge Infrastructure (CNKI). Search strategy based on MeSH terms combining with free text words was applied in English databases, while counterpart terms in Chinese were used in Chinese databases. Besides that, conference literature, as well as related references, have also been screened carefully under the guidance of the snowball strategy, and the language was limited to English or Chinese. When the information we need was incomplete, we contacted the corresponding author to obtain it, and an intentionto-treat (ITT) analysis was also conducted for missing or unreachable data. The full retrieval details are attached in Appendix 1. 


\section{Selection criteria}

The selection criteria were based on the 'PICOS' (participants, intervention, comparator, outcomes, and study designs).

\section{Types of studies}

Only RCTs testing acupuncture for stroke have been enrolled and the language was limited to Chinese or English. Besides that, literature should be available in full papers and the original data should be sufficient and clear. These non-RCTs such as system reviews, case reports, meeting abstracts, clinical experience, and animal trials have been excluded.

\section{Types of participants}

Adults, diagnosed with stroke regardless of ischemic or hemorrhagic stroke by a clear and internationally accepted criterion were eligible and irrespective of nationality, age, gender. However, patients who were medically unstable or unable to follow basic instructions were unqualified.

\section{Types of interventions and comparators}

Non-acupuncture interventions including recommended main treatments such as surgery, drug, rehabilitation or combined with symptomatic and supportive measures were allowed in the control group. Besides the approaches of the control group, acupoint-related therapy including scalpacupuncture, hand-acupuncture, auricular-acupuncture, electro-acupuncture, warm-acupuncture must be applied extra in the experimental group.

\section{Types of outcomes}

At least one of the following outcomes should be included in these studies: Fugl-Meyer assessment (FMA) score and Barthel Index (BI). The former has been broadly used to assess the limb motor function in post-stroke patients $(22,23)$, while the latter can evaluate the activities of daily living (24). In addition, safety assessments including adverse events have been taken into account.

\section{Study screening and data extraction}

First of all, the articles retrieved from these databases were imported into EndNote $\mathrm{X} 7$ to remove duplicate articles automatically, then primary screening was performed independently by two reviewers (Yue Zhuo and Boyu $\mathrm{Wu}$ ) based on titles and abstracts, and trials not meeting the selection criteria were excluded directly. Secondly, these two researchers conducted a full-text screening to select eligible articles, while the exclusion reasons were recorded individually. Thirdly, the reviewers cross-checked the results to ensure the consistency of the screening. When the disagreements appeared, a third senior assessor (Shifeng Deng) was asked to assist in the ultimate judgement.

After completing the above steps, a table for data extraction was established in Microsoft Excel 2016. The main content included: first author's name, nationality, publication year, participants' characteristics, interventions, comparators, and outcomes (i.e., FMA score, BI, and adverse events). Given that baseline situation among different stages of the stroke may be potentially varying, the data finally included in the analysis was estimated by the following formula, which was recommended in the Cochrane Handbook for Systematic Reviews of Interventions (version 5.1) and $r$ is a correlation coefficient with a value of 0.5 (25). This approach allowed for a better presentation of the changes before and after treatment of dissimilar acupuncture intervention time-points.

$$
\bar{x}_{\text {change }}=\bar{x}_{\text {post-treatment }}-\bar{x}_{\text {baseline }}
$$

$$
\begin{aligned}
& S D_{\text {change }}= \\
& \sqrt{\left(S D_{\text {baseline }}\right)^{2}+\left(S D_{\text {post-treatment }}\right)^{2}-2 \times r \times S D_{\text {baseline }} \times S D_{\text {post-treatment }}}
\end{aligned}
$$

\section{Quality assessment}

Two trained researchers (Ming $\mathrm{Xu}$ and Xiaoye Lu) independently assessed the bias risk of all the included RCTs by the Cochrane Collaboration tool (26), which consisted of the following aspects: assignment concealment, random sequence generation, blinding of outcome assessors, blinding of participants and personnel, selective reporting, the integrity of outcome data, and other sources of bias. Each field has been classified as high risk, low risk, or unclear risk. Any disagreements have been resolved by discussion with a third senior assessor (Yuchen Zhang).

\section{Statistical analysis}

First of all, a pairwise meta-analysis was performed using Revman 5.3. (Cochrane Collaboration, Oxford, UK) for the direct comparisons. The heterogeneity of the results was assessed with I-square $\left(\mathrm{I}^{2}\right)$. More specifically, a fixedeffects model was used when $\mathrm{I}^{2}<50 \%$, otherwise a randomeffects model was chosen. As for our study, the results of the 

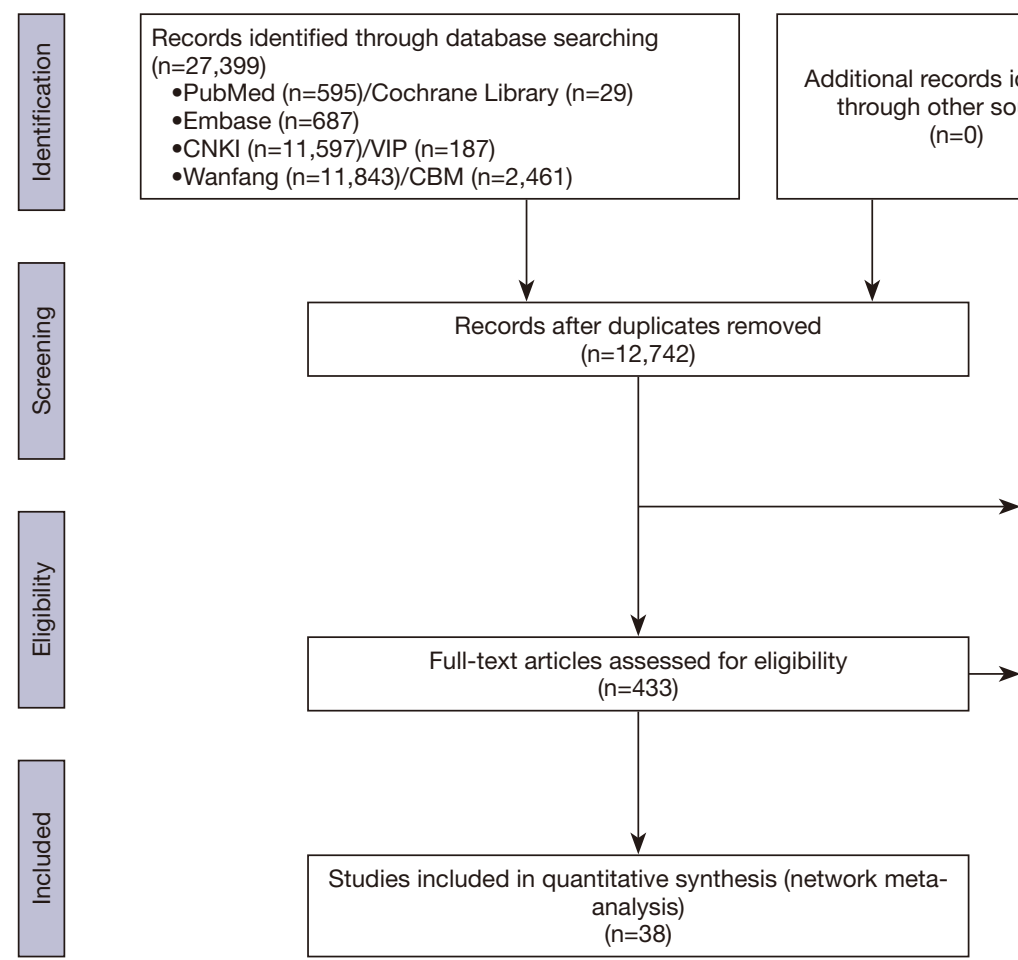

Records excluded afer reading titles and abstracts $(n=12,309)$

Figure 1 Flow chart of study selection.

continuous variable (i.e., FMA score, BI) were reported by mean difference (MD) with $95 \%$ confidence interval (CI).

Secondly, considering the anticipated heterogeneity, the NMA within a Bayesian framework was conducted by WinBUGS 1.4.3 (MRC Biostatistics Unit, Cambridge, UK) based on the random effect model for the results of the indirect comparison. Besides, models were calculated with Markov chain Monte Carlo algorithm (MCMC) (27): four chains were employed for simulation analysis, the step size was set to 10 , the number of annealing times was set to 20,000 for reducing the impact on arbitrary values, and the number of iterations was set to 50,000. Additionally, the continuous outcomes were measured by standard mean difference (SMD) with 95\% CI for indirect comparisons.

Thirdly, the plot of surface under the cumulative ranking (SUCRA) curve was computed by STATA 14.0. (Stata Corporation, College Station, TX, USA) to forecast the possible ranking order (28). In our study, a higher SUCRA score represented the better intervention timepoint for acupuncture in stroke (29). Z-values as well as corresponding $\mathrm{P}$ values were calculated, and $\mathrm{P}$ values less than 0.05 demonstrated a significant difference. Besides, the inconsistency between indirect and direct evidence was assessed by the loop inconsistency test and node-splitting method. Sensitivity analysis was conducted to evaluate the robustness of the results, while publication bias was taken into consideration as well.

\section{Results}

\section{Study search}

As illustrated in Figure 1, a total of 27,399 studies were identified from the above databases based on the established search strategy. After eliminating duplicates, 12,742 studies were left for screening the titles and abstracts. Then, 433 studies were potentially eligible for full-text screening. Finally, 38 trials with 3,836 participants were included in our NMA.

\section{Study description}

Key characteristics of the included studies were listed in Table 1. These studies were published between 1993 and 2020, and most of the participants were from China, while others were from Sweden and Norway, accordingly, 24 studies were written in Chinese and 14 in English. The 
Table 1 Key characteristics of the included studies

\begin{tabular}{|c|c|c|c|c|c|c|c|c|c|c|}
\hline \multirow{2}{*}{ Author, year } & \multirow{2}{*}{ Country } & \multicolumn{2}{|c|}{ Sample size } & \multicolumn{2}{|c|}{ Gender } & \multicolumn{2}{|c|}{ Age: mean \pm SD or range } & \multicolumn{2}{|c|}{ Intervention and time-point } & \multirow{2}{*}{ Outcomes } \\
\hline & & $\mathrm{T}$ & C & $\mathrm{T}(\mathrm{M} / \mathrm{F})$ & $C(\mathrm{M} / \mathrm{F})$ & $\mathrm{T}$ & C & $\mathrm{T}$ & $\mathrm{C}$ & \\
\hline Johansson, 1993 (30) & Sweden & 38 & 40 & \multicolumn{2}{|c|}{$42 / 36$} & $76 \pm N R$ & $75 \pm N R$ & Acu II & Non-Acu II & (2) \\
\hline Kjendahl, 1997 (31) & Norway & 21 & 20 & $16 / 5$ & $15 / 5$ & $35-69$ & $39-72$ & Acu IV & Non-Acu IV & (2) \\
\hline Zhong, 2002 (32) & China & 48 & 48 & \multicolumn{2}{|c|}{$49 / 47$} & NR & NR & Acu III & Non-Acu III & (1)(2) \\
\hline \multirow[t]{2}{*}{ Xie, 2005 (34) } & China & 62 & 60 & $34 / 28$ & $32 / 28$ & $52.0 \pm 6.8$ & $51.8 \pm 7.8$ & Acu I & Acu III & (1)(2) \\
\hline & & 60 & & $31 / 29$ & & $51.4 \pm 7.2$ & & Acu II & & \\
\hline \multirow[t]{2}{*}{ Wang, 2005 (35) } & China & 30 & 30 & \multicolumn{2}{|c|}{$47 / 43$} & \multicolumn{2}{|c|}{$57 \pm 4.5$} & Acu I & Acu III & (1)(2) \\
\hline & & 30 & & & & & & Acu II & & \\
\hline Zhang, 2007 (38) & China & 30 & 30 & $19 / 11$ & $18 / 12$ & $65.23 \pm 11.19$ & $65.26 \pm 11.36$ & Acu II & Non-Acu II & (1)(2) \\
\hline Mao, 2008 (39) & China & 30 & 30 & $17 / 13$ & $15 / 15$ & $66.24 \pm 10.20$ & $65.78 \pm 8.46$ & Acu II & Non-Acu II & (1)(2) \\
\hline \multirow[t]{2}{*}{ Zhang, 2009 (40) } & China & 29 & 29 & $17 / 12$ & $16 / 13$ & $62.93 \pm 8.78$ & $63.62 \pm 6.66$ & Acu II & Non-Acu II & (1)(2)(3) \\
\hline & & 28 & & $15 / 13$ & & $62.79 \pm 6.86$ & & Acu III & & \\
\hline Xue, 2009 (41) & China & 30 & 30 & $19 / 11$ & $16 / 14$ & $56.33 \pm 19.35$ & $53.10 \pm 18.74$ & Acu II & Acu III & (1)(2) \\
\hline Liu, 2010 (42) & China & 45 & 45 & $28 / 17$ & $30 / 15$ & $62.6 \pm 11.4$ & $61.4 \pm 12.5$ & Acu II & Acu III & (1)(2) \\
\hline \multirow[t]{2}{*}{ Li, 2011 (43) } & China & 56 & 52 & $32 / 24$ & $28 / 24$ & $65.8 \pm 10.6$ & $66.7 \pm 10.3$ & Acu I & Non-Acu I & (2) \\
\hline & & 44 & 44 & $24 / 20$ & $23 / 21$ & $65.3 \pm 11.8$ & $66.4 \pm 11.7$ & Acu I & Non-Acu I & (2) \\
\hline Zhang, 2012 (47) & China & 30 & 30 & $20 / 10$ & $17 / 13$ & $18-78$ & $15-78$ & Acu I & Non-Acu I & (2) \\
\hline Zhuang, 2012 (48) & China & 97 & 86 & $63 / 34$ & $54 / 32$ & $64.03 \pm 9.19$ & $64.29 \pm 8.42$ & Acu III & Non-Acu III & (1)(2) (3) \\
\hline Bai, 2013 (49) & China & 40 & 41 & $25 / 15$ & $30 / 11$ & $61.65 \pm 11.05$ & $59.30 \pm 9.66$ & Acu III & Non-Acu III & (1)(2) \\
\hline Gao, 2013 (50) & China & 45 & 16 & $22 / 23$ & $7 / 9$ & $55.3 \pm 5.1$ & $55.9 \pm 5.9$ & Acu I & Non-Acu I & (1) \\
\hline Tong, 2013 (51) & China & 44 & 42 & $25 / 19$ & $22 / 20$ & $69 \pm 6$ & $69 \pm 6$ & Acu III & Non-Acu III & (1) \\
\hline Yang, 2013 (52) & China & 32 & 33 & $17 / 15$ & $17 / 16$ & $64 \pm 9$ & $63 \pm 9$ & Acu IV & Non-Acu IV & (1) \\
\hline Yang, 2014 (53) & China & 33 & 31 & $18 / 15$ & $16 / 15$ & $65 \pm 4$ & $63 \pm 4$ & Acu IV & Non-Acu IV & (2) \\
\hline Zhang, 2014 (54) & China & 30 & 29 & $14 / 16$ & $17 / 12$ & $32-72$ & $32-72$ & Acu IV & Non-Acu IV & (2) \\
\hline Sun, 2015 (55) & China & 33 & 30 & $21 / 12$ & $15 / 15$ & $59 \pm 7$ & $58 \pm 8$ & Acu IV & Non-Acu IV & (2) \\
\hline Wu, 2015 (56) & China & 179 & 174 & $121 / 58$ & $112 / 62$ & $64.92 \pm 11.51$ & $64.51 \pm 11.41$ & Acu IV & Non-Acu IV & (1) \\
\hline
\end{tabular}

Table 1 (continued) 
Table 1 (continued)

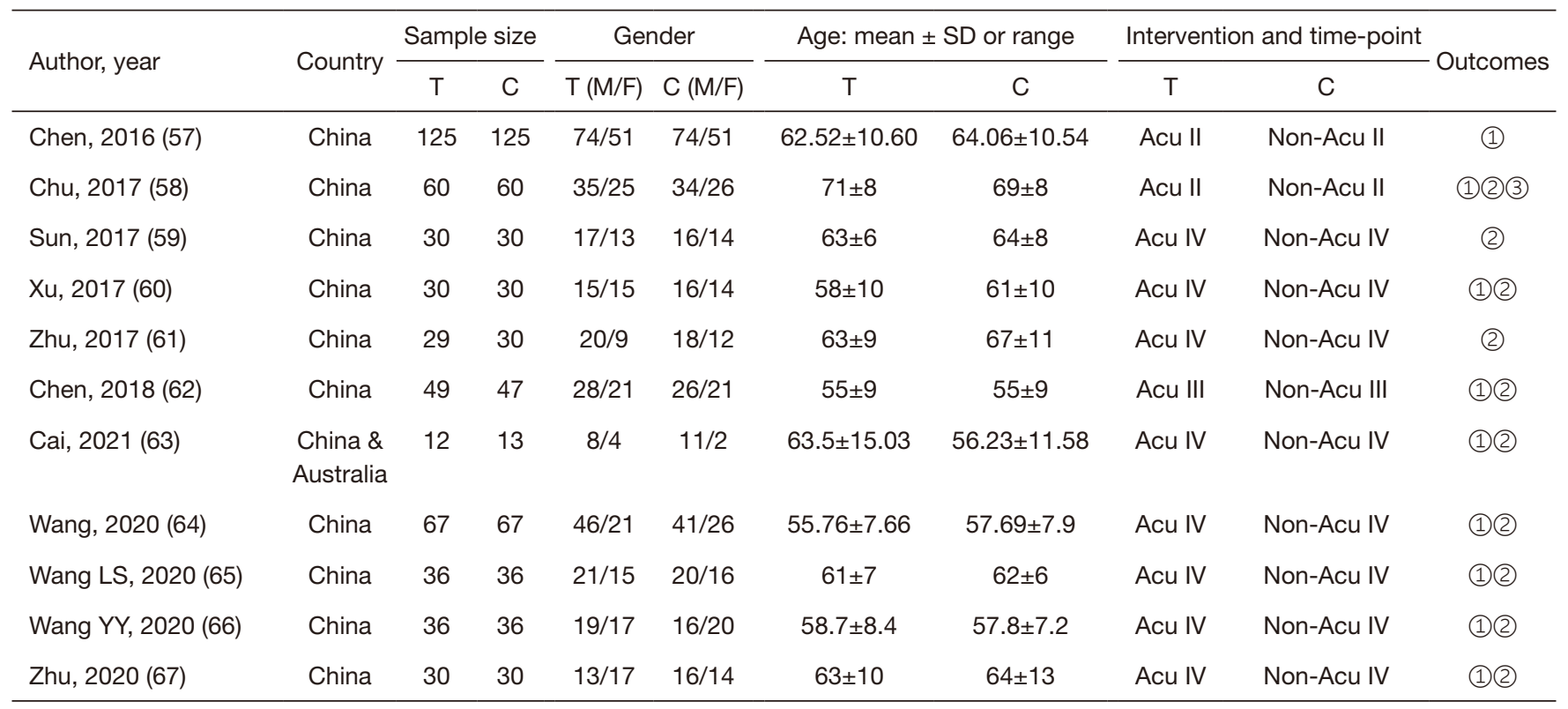

(1) , FMA score; (2) , BI; (3), adverse events; M, male; F, female; T, treatment group; C, control group; Acu I, acupuncture within 48 h poststroke; Acu II, acupuncture within 2-15 d post-stroke; Acu III, acupuncture within 16-30 d post-stroke; Acu IV, acupuncture within 1 month to half a year post-stroke; Non-Acu I, non-acupuncture within $48 \mathrm{~h}$ post-stroke; Non-Acu II, non-acupuncture within 2-15 d post-stroke; Non-Acu III, non-acupuncture within 16-30 d post-stroke; Non-Acu IV, non-acupuncture within 1 month to half a year post-stroke. NR, not reported; FMA, Fugl-Meyer assessment; BI, Barthel Index.

sample size of these studies ranged from 12 to 179 , while the participants' mean ages ranged from 15 to 78 years old. Apart from this, there are 28 of 38 studies reported the FMA score, 34 of 38 studies reported BI, while adverse events were reported in four studies. In addition, the network plot of indirect comparisons was illustrated in Figure 2. To distinguish dissimilar intervention time-points, based on the information from included studies and the actual clinical situation of stroke, we have divided the intervention time points for stroke into four stages: stage I (within $48 \mathrm{~h}$ post-stroke), stage II (within $2-15 \mathrm{~d}$ post-stroke), stage III (within 16-30 d post-stroke), stage IV (within 1 month to half a year post-stroke). Next, depending on the additional application of acupuncture in the experimental group, we have assigned them to eight categories and named them for short as "acupuncture treatment (Acu) I", "Acu II", “Acu III", "Acu IV", "non-acupuncture treatment (Non-Acu) I", "Non-Acu II", "Non-Acu III" and "Non-Acu IV".

\section{Methodological quality assessment}

The risk of bias assessment of the 38 included studies was shown in Figure 3 and Figure 4. Seven studies were judged as high risk for random sequence generation because they were allocated in order of admission. Twenty-six studies didn't describe clearly the method of allocation concealment, which were judged as unclear risk. Considering the features of clinical research in acupuncture, it was hard to conduct a double-blinding method, and informed consent was signed in most of the included studies, thus they were judged as high risk or unclear risk. Because of the drop-out cases, 15 studies were judged as high risk for incomplete outcome data, while the others were low risk. Selective outcome reporting was not commonly found in included studies. In terms of other bias, except for a few high-quality studies with large samples, most of the included studies were difficult to judge accurately.

\section{Pairwise meta-analyses}

\section{FMA score}

As shown in Table 2, we performed 10 direct pairwise meta-analyses to compare the FMA score of dissimilar acupuncture intervention time-points. The FMA score of Acu I showed a greater increase than those of NonAcu I (MD: 20.95; 95\% CI, 11.60 to 30.31); Acu II (MD: 
A

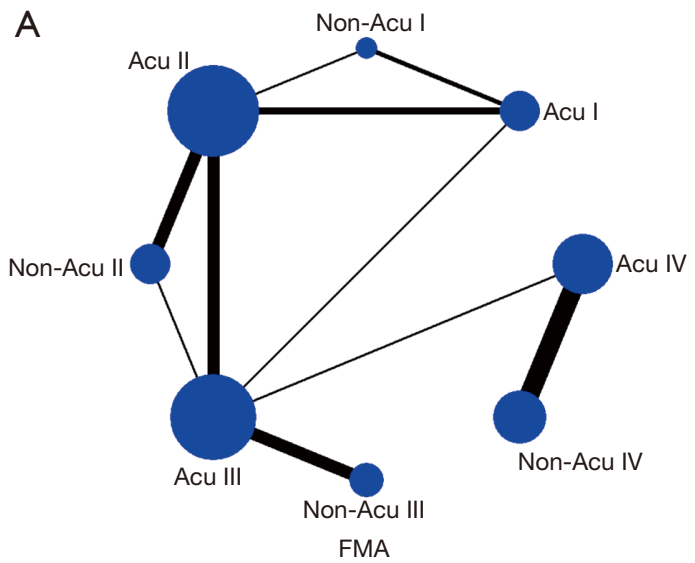

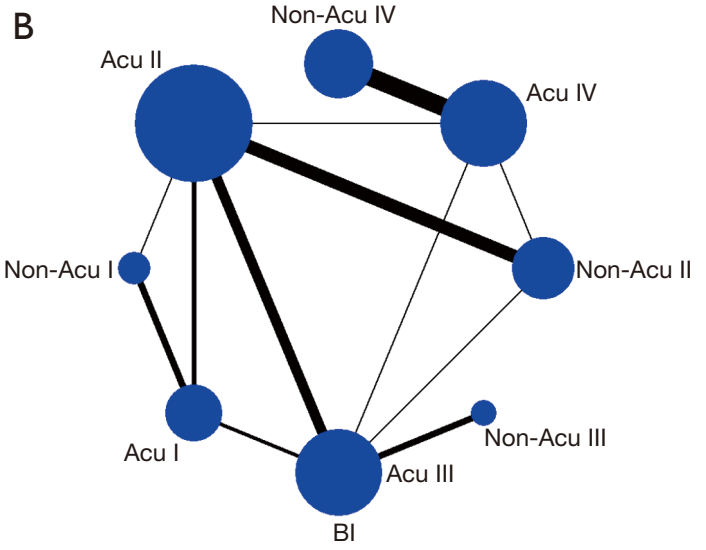

Figure 2 Network plots (A for FMA score, B for BI). Acu I, acupuncture within 48 h post-stroke; Acu II, acupuncture within 2-15 d poststroke; Acu III, acupuncture within 16-30 d post-stroke; Acu IV, acupuncture within 1 month to half a year post-stroke; Non-Acu I, nonacupuncture within 48 h post-stroke; Non-Acu II, non-acupuncture within 2-15 d post-stroke; Non-Acu III, non-acupuncture within 16-30 d post-stroke; Non-Acu IV, non-acupuncture within 1 month to half a year post-stroke. FMA, Fugl-Meyer assessment; BI, Barthel Index.

6.90; 95\% CI, 3.47 to 10.33 ); Acu III (MD: 12.11; $95 \%$ CI, 6.78 to 17.44). Besides, the FMA score of Acu II was significantly higher than those of Non-Acu I (MD: 6.46; 95\% CI, 1.74 to 11.18 ); Non-Acu II (MD: 15.31; 95\% CI, 7.36 to 23.26); Acu III (MD: 14.87; 95\% CI, 7.84 to 21.90). Furthermore, the FMA score of Acu III was significantly higher than those of Non-Acu III (MD: 12.49; 95\% CI, 2.31 to 22.67 ); Acu IV (MD: 6.38; 95\% CI, 3.22 to 9.54). When compared to Non-Acu IV, Acu IV showed a stronger effect in improving FMA score (MD: 9.66; 95\% CI, 5.54 to 13.78). No statistically significant difference between NonAcu II and Acu III (MD: 6.16; 95\% CI, -2.44 to 14.76).

\section{BI}

Similarly, we conducted 10 direct pairwise meta-analyses, which can be seen in Table 2, to compare the changes of BI among dissimilar acupuncture intervention time-points. The BI of Acu I was significantly higher than those of NonAcu I (MD: $11.06 ; 95 \%$ CI, 4.14 to 17.98); Acu II (MD: 6.35; 95\% CI, 0.42 to 12.28 ); Acu III (MD: 12.82 ; $95 \%$ CI, 7.73 to 17.90). Besides, the BI of Acu II showed a greater increase than those of Non-Acu I (MD: 5.43; 95\% CI, 0.74 to 10.39 ); Non-Acu II (MD: 19.55 ; $95 \%$ CI, 16.04 to 23.06); Acu III (MD: 10.55; 95\% CI, 6.54 to 14.56). Furthermore, the BI of Acu III was significantly higher than those of Non-Acu III (MD: 14.27; 95\% CI, 1.46 to 27.08); Acu IV (MD: 7.82; 95\% CI, 4.47 to 11.17). During stage $\mathrm{IV}$, the BI of acupuncture was better than that of Non- acupuncture (MD: 9.78; 95\% CI, 6.91 to 12.64$)$. There was no significant difference between Non-Acu II and Acu III (MD: $6.55 ; 95 \%$ CI, -1.94 to 15.04 ).

\section{$N M A$}

\section{FMA score}

The results of the FMA score were presented in Figure 5, the NMA indicated that Acu I was significantly more efficacious than others. Vary from stage I to IV of stroke, the FMA score of treating without acupuncture was all lower than that of treating with acupuncture (SMD: -19.89; 95\% CI, -26.65 to -13.13 ; SMD: -14.26 ; $95 \%$ CI, -19.10 to -9.42 ; SMD: -11.88 ; $95 \%$ CI, -17.12 to -6.64 ; SMD: -9.76 ; $95 \%$ CI, -13.56 to -5.96$)$. Besides, the earlier acupuncture was applied, the better the FMA score: such as the comparison between Acu I and Acu II (SMD: 7.17; 95\% CI, 1.11 to 13.22); comparison between Acu II and Acu III (SMD: 13.56 ; 95\% CI, 8.79 to 18.33 ); comparison between Acu II and Acu IV (SMD: 19.78; 95\% CI, 8.74 to 30.82). Furthermore, as for the FMA score, our NMA confirmed that it was better to treat early (SMD: $12.72 ; 95 \%$ CI, 2.51 to 22.93 ; SMD: 11.18 ; $95 \%$ CI, 2.90 to 19.46 ; SMD: 15.28; $95 \%$ CI, 2.83 to 27.73 ; SMD: 16.82 ; $95 \%$ CI, 3.07 to 30.57 ), and the combination of acupuncture would be more effective (SMD: 25.44; 95\% CI, 18.38 to 32.50; SMD: 29.54; $95 \%$ CI, 17.87 to 41.21 ; SMD:15.98; $95 \%$ CI, 5.32 to 26.64), additionally, acupuncture was never too late to be 


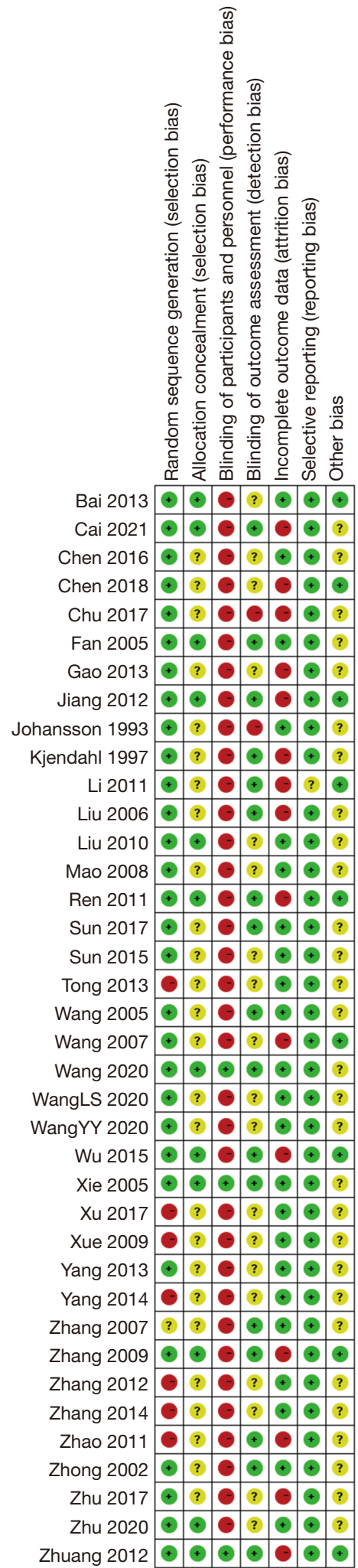

Figure 3 Reviewers' judgements about each risk-of-bias item for included studies. used such as the comparison between Non-Acu I and Acu II (SMD: $-12.72 ; 95 \%$ CI, -20.59 to -4.85 ).

\section{BI}

As shown in Figure 5, the results of BI based on our NMA suggested that Acu I was the most effective. From stage I to IV of stroke, in the view of BI, treating without acupuncture was significantly lower than that of treating with acupuncture (SMD: $-10.84 ; 95 \%$ CI, -16.41 to -5.27 ; SMD: -19.33 ; 95\% CI, -23.17 to -15.49 ; SMD: -10.04 ; 95\% CI, -15.81 to -4.27 ; SMD: -9.69 ; $95 \%$ CI, -12.88 to -6.50). Apart from that, the earlier acupuncture was used, the better the $\mathrm{BI}$, for instance, the comparison between Acu II and Acu III (SMD: 10.28; 95\% CI, 6.14 to 14.42); comparison between Acu II and Acu IV (SMD: 17.99; $95 \%$ CI, 7.15 to 28.83 ). Moreover, from the BI results of NMA, we can conclude that treating early is better (SMD: 13.38; $95 \%$ CI, 5.24 to 21.52 ; SMD: 14.38 ; $95 \%$ CI, 4.74 to 24.02 ; SMD: 21.74 ; $95 \%$ CI, 8.67 to 34.81 ); while the combination of acupuncture would be more effective (SMD: 20.32; $95 \%$ CI, 13.25 to 27.39 ; SMD: 27.68 ; $95 \%$ CI, 16.35 to 39.01 ; SMD: $17.40 ; 95 \% \mathrm{CI}, 6.84$ to 27.96 ), and the comparison between Non-Acu II and Acu III (SMD: -9.04; $95 \%$ CI, -14.52 to -3.56 ) indicated that late application of acupuncture was still helpful sometimes.

\section{Ranking}

The SUCRA-based rankings were displayed in Figure 6. The results showed that, on the aspect of FMA score, Acu I (99.9\%) was the optimal intervention time-point, followed by Acu II (85.8\%), Non-Acu I (57.9\%), Acu III (55.7\%), Non-Acu II (51.5\%), Acu IV (32.1\%), Non-Acu III (13.4\%), Non-Acu IV (3.7\%), which was ranked as the worst. As for BI, Acu I (99.3\%) was still the optimal intervention timepoint, followed by Acu II (85.6\%), Non-Acu I (69.8\%), Acu III (58.1\%), Acu IV (33.5\%), Non-Acu II (27.5\%), NonAcu III (23.4\%), Non-Acu IV (2.8\%), which was ranked as the worst, too.

\section{Inconsistency assessment}

\section{FMA score}

In terms of loop inconsistency test outcome of FMA score, $95 \%$ CI of two loops included 0 , indicating that no significant inconsistency was found. However, one loop (Acu I to Acu II to Non-Acu I) was found statistical inconsistency between indirect and direct comparisons. Apart from this, 


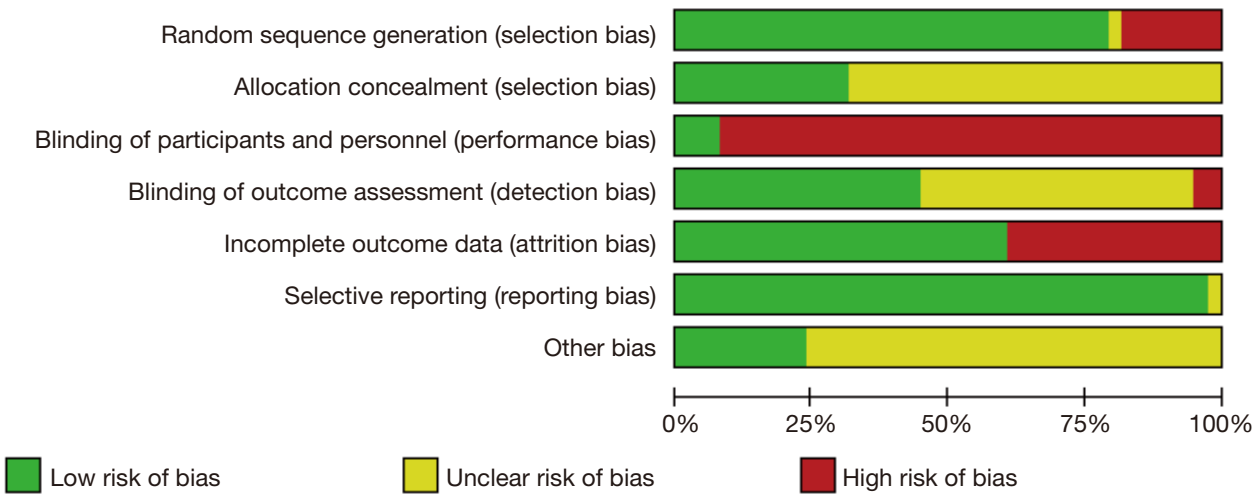

Figure 4 Reviewers' judgements about each risk-of-bias item presented as percentages across all included studies.

the $\mathrm{P}$ value of the global inconsistency test in our NMA was $0.16(\mathrm{P}>0.05)$, and the node-splitting method showed that there were no inconsistencies between direct and indirect comparisons $(\mathrm{P}>0.05)$, except for the comparison between Acu II and Non-Acu I $(\mathrm{P}=0.011<0.05)$.

\section{BI}

For the loop inconsistency test outcome of BI, 95\% CI of five loops included 0 , indicating that no significant inconsistency was found. However, one loop (Acu III to Acu IV to Non-Acu II) was found statistical inconsistency between indirect and direct comparisons. Besides, as for the global inconsistency test, the $\mathrm{P}$ value in our study was $0.92(\mathrm{P}>0.05)$, and the node-splitting method showed that there were no inconsistencies between direct and indirect comparisons (all $\mathrm{P}>0.05$ ). More details were provided in https://cdn.amegroups.cn/static/public/apm-11-1127-1.pdf.

\section{Safety assessment}

Although adverse events were reported in four studies $(36,40,48,58)$, no serious acupuncture-related complications were identified in any of the included studies. Specifically, 10 cases of mild subcutaneous hematoma following an Acu were reported by these four research teams $(36,40,48,58)$, and the hematoma disappeared within a few days. In addition, two cases of syncope during an Acu, which were characterized by dizziness, sweating, and weakness, were reported in two studies $(36,48)$, and the symptoms were relieved promptly with no subsequent physical discomfort after needle withdrawal and resting. Given that most included trials did not record the acupuncture-related adverse events in a standard way, it was hard to conduct a quantitative analysis of safety in our NMA.

\section{Sensitivity analysis and publication bias}

The sensitivity analysis indicated that the results were generally robust (Appendix 2), while no evidence of publication bias was revealed in our funnel plots (Appendix 3).

\section{Discussion}

A considerable number of people suffer from post-stroke motor dysfunction as well as impairment in the activities of daily living around the world (2). Acupuncture has been broadly applied to post-stroke patients as a complementary and alternative therapy in East Asian countries, especially in China, for many years (68). The advantages of acupuncture include safety, reliability, and easy operation. As a result, the experimental and clinical research on acupuncture in treating stroke has been increasing significantly in recent years, and most results have been promising (69). However, most studies have focused on how to make acupuncture more effective or the mechanisms by which it works, while many of these studies have not been uniform or standardized in their design and the outcomes always stay in a low level of evidence. As for stroke, the intervention time-point for acupuncture in different trials is nonuniform, which indicates there is no unified consensus on this issue, further, it still confuses clinical choices. Although, some related studies have been conducted in this area $(70,71)$, the comparisons are limited in scope, and more importantly, there is a lack of evidence based on Bayesian NMA, which can combine direct and indirect comparisons 
Table 2 Pairwise meta-analyses

\begin{tabular}{|c|c|c|c|c|c|}
\hline Comparison & Pairwise or $(95 \% \mathrm{Cl})$ & Number of patients & Number of studies & \multicolumn{2}{|c|}{ Heterogeneity test } \\
\hline \multicolumn{6}{|l|}{ FMA } \\
\hline Acu III vs. Non-Acu III & $12.49(2.31,22.67)$ & 543 & 5 & 91 & 0.02 \\
\hline Acu II vs. Non-Acu II & $15.31(7.36,23.26)$ & 532 & 5 & 94 & 0.0002 \\
\hline Acu IV vs. Non-Acu IV & $9.66(5.54,13.78)$ & 841 & 8 & 88 & $<0.00001$ \\
\hline Acu II vs. Acu III & $14.87(7.84,21.90)$ & 365 & 5 & 92 & $<0.0001$ \\
\hline Acu I vs. Acu II & $6.90(3.47,10.33)$ & 258 & 3 & 46 & $<0.0001$ \\
\hline Acu III vs. Acu IV & $6.38(3.22,9.54)$ & 55 & 1 & - & - \\
\hline Acu II vs. Non-Acu I & $6.46(1.74,11.18)$ & 40 & 1 & - & - \\
\hline \multicolumn{6}{|l|}{$\mathrm{BI}$} \\
\hline Acu II vs. Non-Acu II & $19.55(16.04,23.06)$ & 808 & 8 & 90 & $<0.00001$ \\
\hline Acu I vs. Non-Acu I & $11.06(4.14,17.98)$ & 295 & 4 & 77 & 0.002 \\
\hline Acu I vs. Non-Acu IV & $9.78(6.91,12.64)$ & 769 & 12 & 79 & $<0.00001$ \\
\hline Acu III vs. Non-Acu III & $14.27(1.46,27.08)$ & 456 & 4 & 94 & 0.03 \\
\hline Acu II vs. Acu III & $10.55(6.54,14.56)$ & 425 & 6 & 84 & $<0.00001$ \\
\hline Acu I vs. Acu II & $6.35(0.42,12.28)$ & 220 & 3 & 73 & 0.04 \\
\hline
\end{tabular}

Acu I, acupuncture within $48 \mathrm{~h}$ post-stroke; Acu II, acupuncture within 2-15 d post-stroke; Acu III, acupuncture within 16-30 d poststroke; Acu IV, acupuncture within 1 month to half a year post-stroke; Non-Acu I, non-acupuncture within 48 h post-stroke; Non-Acu II, non-acupuncture within 2-15 d post-stroke; Non-Acu III, non-acupuncture within 16-30 d post-stroke; Non-Acu IV, non-acupuncture within 1 month to half a year post-stroke. FMA, Fugl-Meyer assessment; BI, Barthel Index.

across multi-dimensions. Thus, the purpose of our NMA is to identify the efficacy and safety of dissimilar acupuncture intervention time-points for stroke, especially in motor function and the activities of daily living, then confirm whether acupuncture is helpful in stroke, if so, whether the early application is better than late. To our knowledge, this study is the first attempt in this field.

In our NWA, the association of Acus and Non-Acus with dissimilar acupuncture intervention time-points for improving stroke-related impairments was compared by the combination of indirect and direct evidence from 38
RCTs in 3,836 participants. To avoid the impact resulting from the process of stroke recovery on our data analysis, we included the Non-Acu group from each of the same studies as a comparator, while also allowing us to compare the efficacy and safety of Acu and Non-Acu. Meanwhile, the classification of intervention time-point was based on the commonalities among included studies as well as the actual clinical situation of stroke.

Four important findings have been obtained in the end. First, acupuncture is effective in improving capacity in motor function as well as the activities of daily living after 


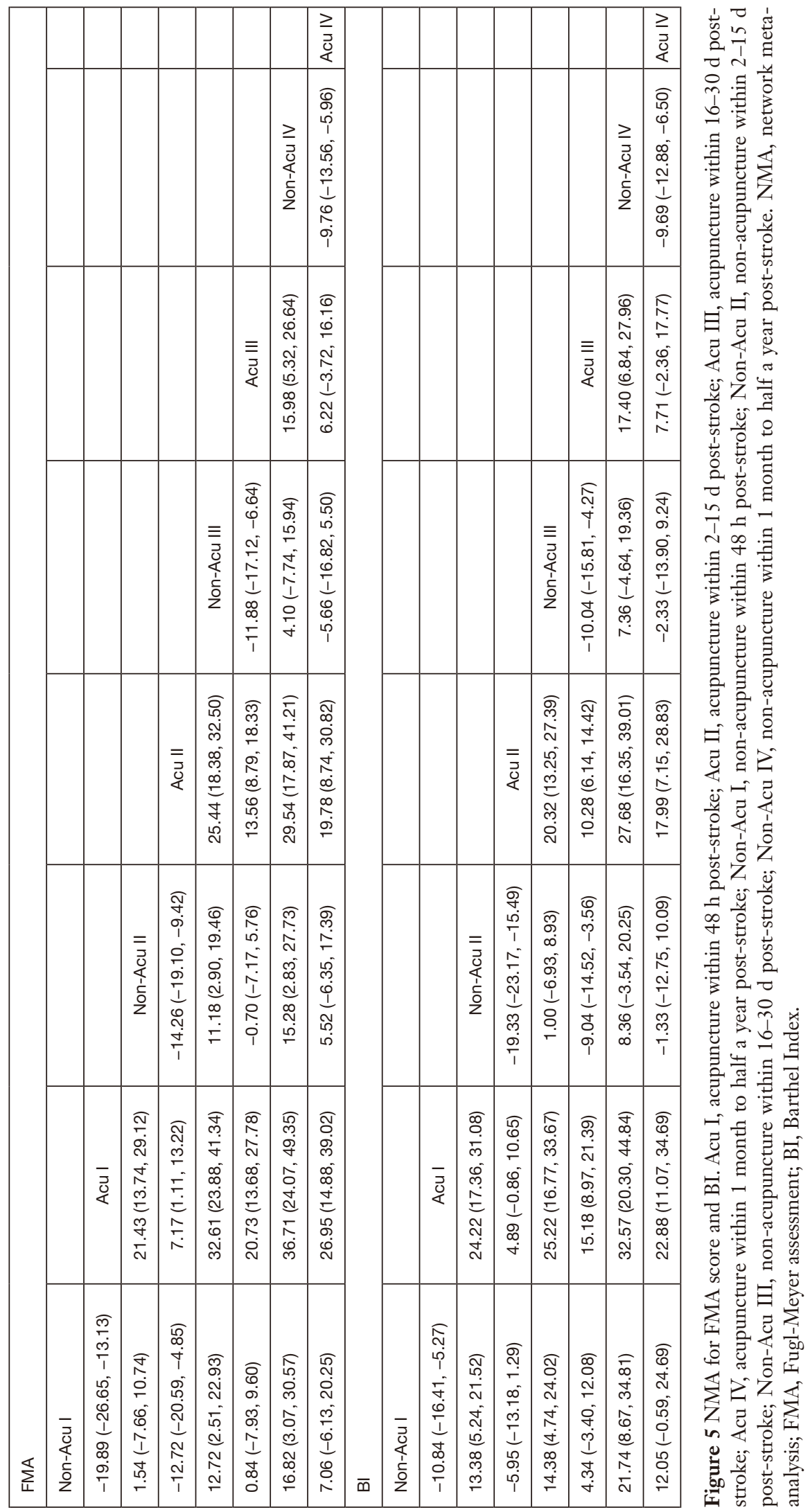


A
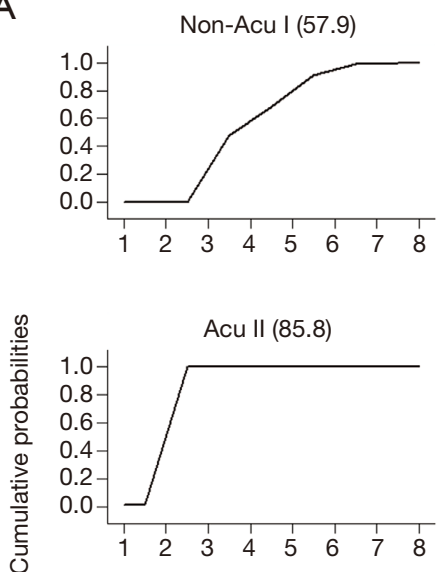

Non-Acu IV (3.7)

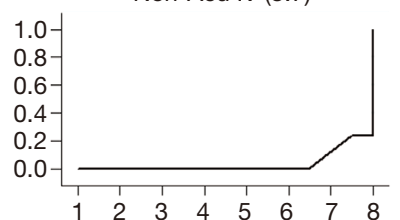

Fugl-meyer assessment

\section{B}
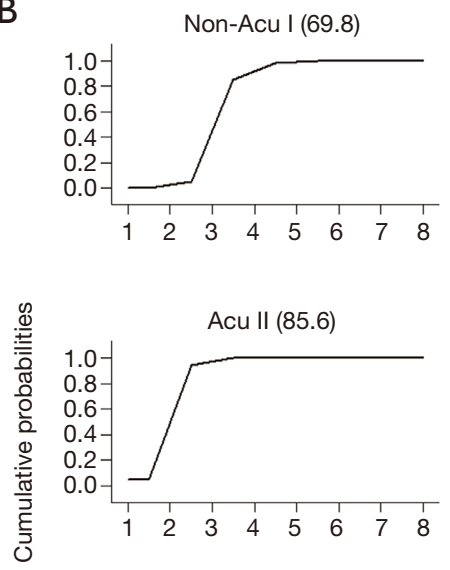

Non-Acu IV (2.8)

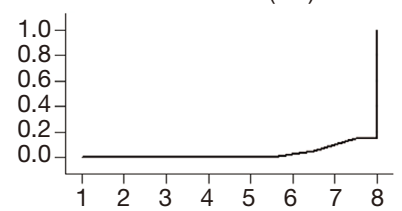

Barthel index
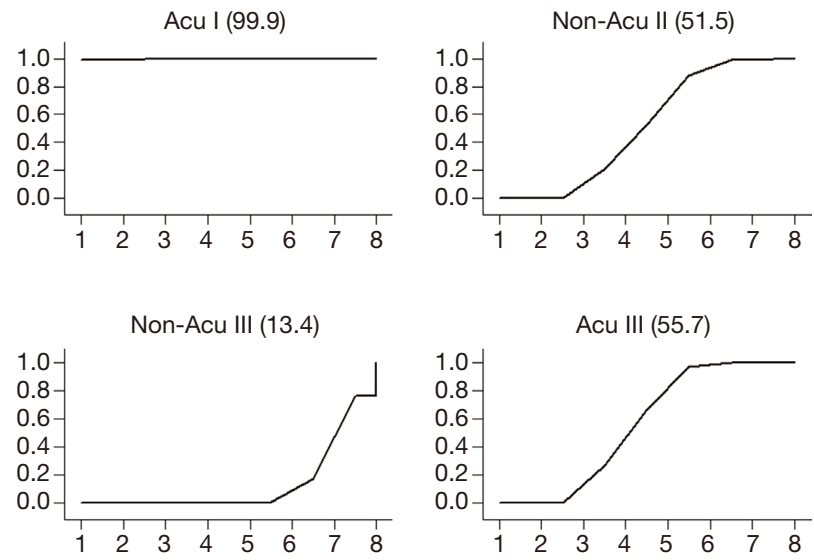

Acu IV (32.1)
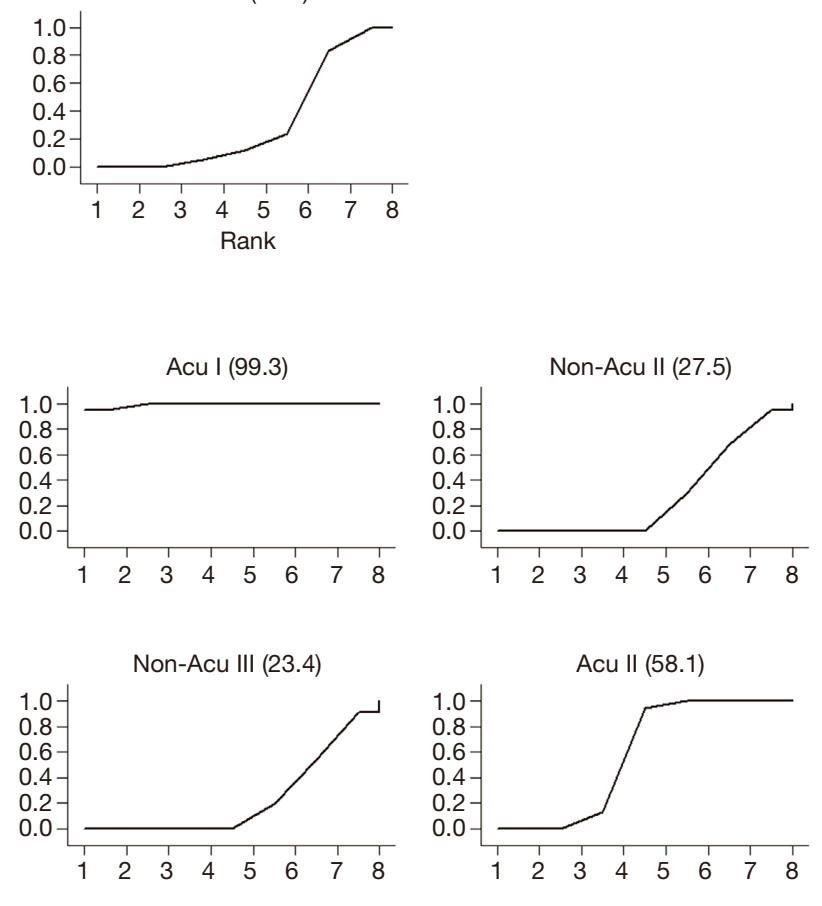

Acu IV (33.5)

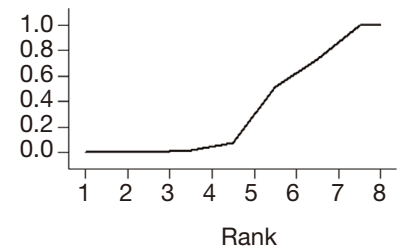

Figure 6 SUCRA curves (A for FMA score, B for BI). Acu I, acupuncture within 48 h post-stroke; Acu II, acupuncture within 2-15 d poststroke; Acu III, acupuncture within 16-30 d post-stroke; Acu IV, acupuncture within 1 month to half a year post-stroke; Non-Acu I, nonacupuncture within 48 h post-stroke; Non-Acu II, non-acupuncture within 2-15 d post-stroke; Non-Acu III, non-acupuncture within 16$30 \mathrm{~d}$ post-stroke; Non-Acu IV, non-acupuncture within 1 month to half a year post-stroke. SUCRA, surface under the cumulative ranking; FMA, Fugl-Meyer assessment; BI, Barthel Index. 
stroke with good safety. Second, at similar baseline levels or course of the stroke, compared to Non-Acus, a combination of treatments including acupuncture can deliver greater benefits to patients and mitigate their sequelae. In other words, acupuncture can be helpful for patients with stroke regardless of disease duration. Third, we can conclude from the ranking graphs that earlier intervention for stroke is better than later with a course-dependent decreased levels of improvement of FMA and BI score by acupuncture. In brief, the earlier acupuncture is used, the better the prognosis of stroke. Fourth, the optimal acupuncture intervention time-point for stroke is within $48 \mathrm{~h}$ poststroke, and the significant validity period lasts until 15 days after onset.

In previous studies and guidelines on stroke, whether acupuncture should be applied as soon as possible is still controversial, and some scholars believe that acupuncture should only be used at the end of clinical treatment or the beginning of functional rehabilitation. However, the notion of early rehabilitation for stroke has been increasingly accepted (72). It has been reported that early rehabilitation not only promotes better neurological recovery and regulates central neural excitability $(73,74)$, but also improves blood circulation in the affected limb and facilitates the recuperation of motor function as well as the emergence of normal movement patterns, thereby enhancing the patient's ability to perform daily activities (75). From the pathology perspective, myogenic fibers begin to shorten or atrophy after 3 days of hemiparesis and after 2 weeks, the dense connective tissue hyperplasia appears around the joint. Some studies have found that when the vital signs and neurological status stabilized, 24-48 h post-stroke is regarded as the optimal time window to conduct a combined rehabilitation of passive and active methods (76-78), while clinical gains began to diminish $14 \mathrm{~d}$ post-stroke (79). Obviously, our findings are largely consistent with it. Acupuncture, an alternative passive rehabilitation method, combined with active functional exercise can better facilitate the resumption of motor function and activities of daily living (80). In addition, based on the plasticity and functional reorganization of the nervous system, early intervention of acupuncture can accelerate the establishment of cerebral collateral circulation, increase cerebral blood flow, improve cerebral oxygen metabolism, protect central neurons, reduce the apoptosis of cerebral neurons, promote the reorganization and compensation of peri-lesion tissue or healthy brain cells (81-84). In brief, it can better exploit neuroplasticity. On the contrary, since neurons are difficult to regenerate, late application of acupuncture can be less effective, even though it is still effective.

There are several attractive advantages of this NMA. Although cerebral infarction and hemorrhage are pathologically distinct, they have a lot in common in terms of rehabilitation and Acu, thereby expanding the scope of the study. Furthermore, we performed an all-around search and included all qualified studies to compare the efficacy of dissimilar intervention time-points among Acus and Non-Acus under a Bayesian framework. What's more, the rank test of effectiveness provides data to favor acupuncture as well as early application, which may lead to publication bias.

However, there are still some limitations to our study. Firstly, due to a limitation of languages and most included studies were conducted in China among Chinese populations, selection bias was inevitable, which may limit the generalization of our findings. Secondly, to some extent, positive results tend to be preferred by acupuncture practitioners, which increase the risk of publication bias. Thirdly, it was hard for us to rule out heterogeneity because of the difference in acupoint selections, acupuncture techniques, and treatment frequency. Fourthly, owing to poor reporting, most included studies were judged as having an unclear risk of bias in terms of blinding, allocation concealment, which may lower the credibility of our findings to some extent. Finally, due to lack of followup duration data in many included RCTs, unaddressed concerns still exist regarding the long-term impacts of treating stroke with acupuncture. All the above issues need to be addressed in the future to improve the quality of acupuncture research.

\section{Conclusions}

Because of the poor quality of the included studies, the credibility of our conclusions is low, however, our NMA indicate that treatments with acupuncture are more helpful in improving motor function and the activities of daily living after stroke with good safety, what's more, the soon use of acupuncture the better the efficacy. The optimal acupuncture intervention time-point for stroke is within $48 \mathrm{~h}$ post-stroke and the significant validity period lasts until 15 days after onset. Besides, well-designed studies on acupuncture with high quality and large samples are greatly needed in the future. 


\section{Acknowledgments}

Funding: This work was supported by the National Natural Science Foundation of China (No.81473753, No. 81173327); The Integrative Medicine Open Fund Project of "Domestic First-Class Training Discipline" of Hunan Province (2020ZXYJH37); Changsha Municipal Natural Science Foundation (kq2007041); The Scientific Research Project of Department of Education of Hunan Province (No. 18C0367).

\section{Footnote}

Reporting Checklist: The authors have completed the PRISMA-NMA reporting checklist. Available at https:// dx.doi.org/10.21037/apm-21-1127

Conflicts of Interest: All authors have completed the ICMJE uniform disclosure form (available at https://dx.doi. org/10.21037/apm-21-1127). The authors have no conflicts of interest to declare.

Ethical Statement: The authors are accountable for all aspects of the work in ensuring that questions related to the accuracy or integrity of any part of the work are appropriately investigated and resolved.

Open Access Statement: This is an Open Access article distributed in accordance with the Creative Commons Attribution-NonCommercial-NoDerivs 4.0 International License (CC BY-NC-ND 4.0), which permits the noncommercial replication and distribution of the article with the strict proviso that no changes or edits are made and the original work is properly cited (including links to both the formal publication through the relevant DOI and the license). See: https://creativecommons.org/licenses/by-nc-nd/4.0/.

\section{References}

1. Kim J, Thayabaranathan T, Donnan GA, et al. Global Stroke Statistics 2019. Int J Stroke 2020;15:819-38.

2. Lindsay MP, Norrving B, Sacco RL, et al. World Stroke Organization (WSO): Global Stroke Fact Sheet 2019. Int J Stroke 2019;14:806-17.

3. Wu S, Wu B, Liu M, et al. Stroke in China: advances and challenges in epidemiology, prevention, and management. Lancet Neurol 2019;18:394-405.

4. Katan M, Luft A. Global Burden of Stroke. Semin Neurol
2018;38:208-11.

5. Jauch EC, Saver JL, Adams HP Jr, et al. Guidelines for the early management of patients with acute ischemic stroke: a guideline for healthcare professionals from the American Heart Association/American Stroke Association. Stroke 2013;44:870-947.

6. van der Meij A, Wermer MJH. Vagus nerve stimulation: a potential new treatment for ischaemic stroke. Lancet 2021;397:1520-1.

7. Elsner B, Kwakkel G, Kugler J, et al. Transcranial direct current stimulation ( $\mathrm{tDCS}$ ) for improving capacity in activities and arm function after stroke: a network metaanalysis of randomised controlled trials. J Neuroeng Rehabil 2017;14:95.

8. Yang L, Tan JY, Ma H, et al. Warm-needle moxibustion for spasticity after stroke: A systematic review of randomized controlled trials. Int J Nurs Stud 2018;82:129-38.

9. Chen LY, Yen HR, Sun MF, et al. Acupuncture treatment is associated with a decreased risk of developing stroke in patients with depression: A propensity-score matched cohort study. J Affect Disord 2019;250:298-306.

10. Liu AJ, Li JH, Li HQ, et al. Electroacupuncture for Acute Ischemic Stroke: A Meta-Analysis of Randomized Controlled Trials. Am J Chin Med 2015;43:1541-66.

11. Zhu Y, Yang Y, Li J. Does acupuncture help patients with spasticity? A narrative review. Ann Phys Rehabil Med 2019;62:297-301.

12. Zhang $\mathrm{S}, \mathrm{Wu} \mathrm{B}, \mathrm{Liu} \mathrm{M}$, et al. Acupuncture efficacy on ischemic stroke recovery: multicenter randomized controlled trial in China. Stroke 2015;46:1301-6.

13. Gosman-Hedström G, Claesson L, Klingenstierna U, et al. Effects of acupuncture treatment on daily life activities and quality of life: a controlled, prospective, and randomized study of acute stroke patients. Stroke 1998;29:2100-8.

14. Li HQ, Li Y, Chen ZX, et al. Electroacupuncture Exerts Neuroprotection through Caveolin-1 Mediated Molecular Pathway in Intracerebral Hemorrhage of Rats. Neural Plast 2016;2016:7308261.

15. Chavez LM, Huang SS, MacDonald I, et al. Mechanisms of Acupuncture Therapy in Ischemic Stroke Rehabilitation: A Literature Review of Basic Studies. Int J Mol Sci 2017;18:2270.

16. Yang A, Wu HM, Tang JL, et al. Acupuncture for stroke rehabilitation. Cochrane Database Syst Rev 2016;(8):CD004131.

17. Wu P, Mills E, Moher D, et al. Acupuncture in poststroke rehabilitation: a systematic review and meta-analysis of randomized trials. Stroke 2010;41:e171-9. 
18. Jiang C, Yang S, Tao J, et al. Clinical Efficacy of Acupuncture Treatment in Combination With RehaCom Cognitive Training for Improving Cognitive Function in Stroke: A 2×2 Factorial Design Randomized Controlled Trial. J Am Med Dir Assoc 2016;17:1114-22.

19. Mulla SM, Wang L, Khokhar R, et al. Management of Central Poststroke Pain: Systematic Review of Randomized Controlled Trials. Stroke 2015;46:2853-60.

20. Shen PF, Kong L, Ni LW, et al. Acupuncture intervention in ischemic stroke: a randomized controlled prospective study. Am J Chin Med 2012;40:685-93.

21. Hutton B, Salanti G, Caldwell DM, et al. The PRISMA extension statement for reporting of systematic reviews incorporating network meta-analyses of health care interventions: checklist and explanations. Ann Intern Med 2015;162:777-84.

22. Sullivan KJ, Tilson JK, Cen SY, et al. Fugl-Meyer assessment of sensorimotor function after stroke: standardized training procedure for clinical practice and clinical trials. Stroke 2011;42:427-32.

23. Gladstone DJ, Danells CJ, Black SE. The fugl-meyer assessment of motor recovery after stroke: a critical review of its measurement properties. Neurorehabil Neural Repair 2002;16:232-40.

24. Quinn TJ, Langhorne P, Stott DJ. Barthel index for stroke trials: development, properties, and application. Stroke 2011;42:1146-51.

25. Abrams KR, Gillies CL, Lambert PC. Meta-analysis of heterogeneously reported trials assessing change from baseline. Stat Med 2005;24:3823-44.

26. Higgins JP, Altman DG, Gøtzsche PC, et al. The Cochrane Collaboration's tool for assessing risk of bias in randomised trials. BMJ 2011;343:d5928.

27. Ades AE, Sculpher M, Sutton A, et al. Bayesian methods for evidence synthesis in cost-effectiveness analysis. Pharmacoeconomics 2006;24:1-19.

28. Salanti G, Ades AE, Ioannidis JP. Graphical methods and numerical summaries for presenting results from multipletreatment meta-analysis: an overview and tutorial. J Clin Epidemiol 2011;64:163-71.

29. Chaimani A, Higgins JP, Mavridis D, et al. Graphical tools for network meta-analysis in STATA. PLoS One 2013;8:e76654.

30. Johansson K, Lindgren I, Widner H, et al. Can sensory stimulation improve the functional outcome in stroke patients? Neurology 1993;43:2189-92.

31. Kjendahl A, Sällström S, Osten PE, et al. A one year follow-up study on the effects of acupuncture in the treatment of stroke patients in the subacute stage: a randomized, controlled study. Clin Rehabil 1997;11:192-200.

32. Zhong CM, Liu QF, Jin HY, et al. Effects of acupuncture and balance facilitation of muscular tension on the early rehabilitation of patients with stroke and hemiplegia. Chinese Journal of Clinical Rehabilitation 2002;23:3612-3.

33. Fan YY, Zhou DX, Zhang TD, et al. Clinical study of acupuncture and rehabilitation for post-stroke motor dysfunction in different time-points. Central Plains Medical Journal 2005;32:5-6.

34. Xie QF, Wang JH, Zou YH, et al. Acupuncture therapy at three different time for motor function and activity of daily life in patients with acute cerebral infarction. Chinese Journal of Clinical Rehabilitation 2005;(17):128-9.

35. Wang JF, Jin Q, Xie QF. Clinical observation of the timing of intervention by acupuncture in the acute phase of cerebral hemorrhage. Journal of Beijing University of Traditional Chinese Medicine 2005;(3):72-4.

36. Liu H, Wang Y, Ren H, et al. An effect of acupuncture on ADL and cognitive function in patients with ischemic stroke. Chinese Journal of Rehabilitation Medicine 2006;21:444-8.

37. Wang XY. Effects of the improved acupoints and rehabilitation exercise on locomotor ability of the upper limbs and ability of daily life in the patient of cerebral infarction. Zhongguo Zhen Jiu 2007;27:179-81.

38. Zhang W, Feng L, Jia X, et al. Clinical observation on therapeutic effects of the point-penetrating method in acupuncture treatment of spastic hemiparalysis due to cerebrovascular disorders. J Tradit Chin Med 2007;27:170-2.

39. Mao M, Chen X, Chen Y, et al. Stage-oriented comprehensive acupuncture treatment plus rehabilitation training for apoplectic hemiplegia. J Tradit Chin Med 2008;28:90-3

40. Zhang SS. The influences of two different intervention periods of acupuncture therapy on limb motor function in patients with cerebral infarction. Guangzhou University of Chinese Medicine 2009;21-5.

41. Xue FL, Li HP. Comparison clinical effect between early and no early of acupuncture treatment with rehabilitative exercises of stroke. Modern Medicine Journal of China 2009;11:19-21.

42. Liu WA, Wu QM, Li XR, et al. The effect of different periods of interventional acupuncture and rehabilitation on stroke patients with hemiplegia. Chinese Journal of Information on TCM 2010;17:60-1. 
43. Li JY, Zhao Y, Zhang ZN. Impacts of Tongnao Huoluo acupuncture therapy on BI and NIHSS of patients with acute cerebral infraction. Zhongguo Zhong Xi Yi Jie He Za Zhi 2011;31:28-32.

44. Zhao ZE, Cai J, Deng SP. Clinical observation of early intervention acupuncture with rehabilitation training for hemiplegia in stroke. Jilin Medical Journal 2011;32:5303-4.

45. Ren YJ. Investigation on the effect of acupuncture and rehabilitation therapy in patients with acute cerebral infarction of different periods and the study of fMRI. Nanjing University of Chinese Medicine 2011;20-1.

46. Jiang $M$. The chronergy study of acupuncture combined with rehabilitation training in the treatment of ischemic stroke. Guangzhou University of Chinese Medicine 2012;26-7.

47. Zhang M. Clinical research on traumatic cerebral infarction treated with mild-hypothermia and acupuncture. Zhongguo Zhen Jiu 2012;32:697-700.

48. Zhuang LX, Xu SF, D'Adamo CR, et al. An effectiveness study comparing acupuncture, physiotherapy, and their combination in poststroke rehabilitation: a multicentered, randomized, controlled clinical trial. Altern Ther Health Med 2012;18:8-14.

49. Bai YL, Li L, Hu YS, et al. Prospective, randomized controlled trial of physiotherapy and acupuncture on motor function and daily activities in patients with ischemic stroke. J Altern Complement Med 2013;19:684-9.

50. Gao H, Li X, Gao X, et al. Contralateral needling at unblocked collaterals for hemiplegia following acute ischemic stroke. Neural Regen Res 2013;8:2914-22.

51. Tong S, Su L, Lü HB, et al. Observation on the efficacy of acupuncture at key acupoints combined with rehabilitation therapy for spasmodic hemiplegia after cerebral infarction. Zhongguo Zhen Jiu 2013;33:399-402.

52. Yang HT, Zhuang LX, Liu Y. Efficacy observation on post-stroke spastic hemiplegia treated with temporal threeneedle and spastic three-needle therapy. Zhongguo Zhen Jiu 2013;33:889-92.

53. Yang XH, Liu B, OuYang BS. Effect of acupuncture combined with rehabilitative training on neural functional recovery of stroke patients during recovery phase: a randomized controlled trial. World Journal of Acupuncture-Moxibustion 2014;24:17-23.

54. Zhang HY, Li PF. Observation on efficacy of acupuncture combined with rehabilitation training for post-stroke balance disorders. World Journal of Acupuncture-
Moxibustion 2014;24:25-9.

55. Sun P, Chu H, Li P, et al. The effect of the acupuncture intervention of dredging Governor Vessel and regulating mentality for the medication treatment of post-stroke depression. Zhongguo Zhen Jiu 2015;35:753-7.

56. Wu XL, Mi ZP, Wang HS, et al. Effect of Acupuncture Combined Physical Training and Relearning on Stroke Rehabilitation: a Multi-center Randomized Controlled Clinical Study. Zhongguo Zhong Xi Yi Jie He Za Zhi 2015;35:549-54.

57. Chen L, Fang J, Ma R, et al. Additional effects of acupuncture on early comprehensive rehabilitation in patients with mild to moderate acute ischemic stroke: a multicenter randomized controlled trial. BMC Complement Altern Med 2016;16:226.

58. Chu H, Zhang S, Fu J, et al. TIE's flying acupuncture for acute cerebral infarction hemiplegia: a randomized controlled trial. Zhongguo Zhen Jiu 2017;37:1153-6.

59. Sun R, Tian L, Fang X, et al. Clinical study of post-stroke upper limb spasmodic hemiplegia treated with jingou diaoyu needling technique and Bobath therapy. Zhongguo Zhen Jiu 2017;37:372-6.

60. Xu L, Wang M, Li F, et al. Acupuncture combined with rehabilitation training for the limb spasm after stroke. Zhongguo Zhen Jiu 2017;37:696-700.

61. Zhu F, Gao J, Gao R, et al. Clinical efficacy of electroacupuncture combined with motor imagery therapy on hemiplegic cerebral infarction. Zhongguo Zhen Jiu 2017;37:927-31.

62. Chen Y, Zhou H, Jin T, et al. Clinical observation of the phased acupuncture for ischemic stroke hemiplegia. Zhongguo Zhen Jiu 2018;38:1027-34.

63. Cai Y, Zhang CS, Zhang AL, et al. Electroacupuncture for Poststroke Spasticity: Results of a Pilot Pragmatic Randomized Controlled Trial. J Pain Symptom Manage 2021;61:305-14.

64. Wang HQ, Hou M, Li H, et al. Effects of acupuncture treatment on motor function in patients with subacute hemorrhagic stroke: A randomized controlled study. Complement Ther Med 2020;49:102296.

65. Wang LS, Li GN, Gu XT. Effects of pricking blood therapy at meridian sinews on upper limb spasm of post-stroke patients. World Journal of Acupuncture-Moxibustion 2020;30:39-44.

66. Wang YY, He L, Ye JB, et al. Clinical effect of plumblossom needle tapping at three yin meridians of wrist on wrist joint contracture after stroke. Zhongguo Zhen Jiu 2020;40:26-9. 
67. Zhu JM, Zhuang R, He J, et al. Yin-yang balance penetrating acupuncture combined with rehabilitation training on upper limb spasticity in stroke hemiplegia. Zhongguo Zhen Jiu 2020;40:697-701.

68. Luo Y, Wang CZ, Hesse-Fong J, et al. Application of Chinese Medicine in Acute and Critical Medical Conditions. Am J Chin Med 2019;47:1223-35.

69. Zhu W, Ye Y, Liu Y, et al. Mechanisms of Acupuncture Therapy for Cerebral Ischemia: an Evidence-Based Review of Clinical and Animal Studies on Cerebral Ischemia. J Neuroimmune Pharmacol 2017;12:575-92.

70. Teasell R, Mehta S, Pereira S, et al. Time to rethink longterm rehabilitation management of stroke patients. Top Stroke Rehabil 2012;19:457-62.

71. Molchanova EE, Drobyshev VA. Acupuncture as a factor of correction of «final points» and improving the quality of life at the stage of rehabilitation of patients with ischemic stroke. Vopr Kurortol Fizioter Lech Fiz Kult 2020;97:11-6.

72. Coleman ER, Moudgal R, Lang K, et al. Early Rehabilitation After Stroke: a Narrative Review. Curr Atheroscler Rep 2017;19:59.

73. Luo CX, Jiang J, Zhou QG, et al. Voluntary exerciseinduced neurogenesis in the postischemic dentate gyrus is associated with spatial memory recovery from stroke. J Neurosci Res 2007;85:1637-46.

74. Zhang L, Hu X, Luo J, et al. Physical exercise improves functional recovery through mitigation of autophagy, attenuation of apoptosis and enhancement of neurogenesis after MCAO in rats. BMC Neurosci 2013;14:46.

75. Chippala P, Sharma R. Effect of very early mobilisation on functional status in patients with acute stroke: a single-blind, randomized controlled trail. Clin Rehabil 2016;30:669-75.

Cite this article as: Zhuo Y, Xu M, Deng S, Zhang Y, Lu X, Wu B, Zhang H. Efficacy and safety of dissimilar acupuncture intervention time-points in treating stroke: a systematic review and network meta-analysis. Ann Palliat Med 2021;10(10):1019610212. doi: 10.21037/apm-21-1127
76. Zhang P, Xianglei J, Hongbo Y, et al. Neuroprotection of Early Locomotor Exercise Poststroke: Evidence From Animal Studies. Can J Neurol Sci 2015;42:213-20.

77. Zheng HQ, Zhang LY, Luo J, et al. Physical exercise promotes recovery of neurological function after ischemic stroke in rats. Int J Mol Sci 2014;15:10974-88.

78. Yang YR, Wang RY, Wang PS. Early and late treadmill training after focal brain ischemia in rats. Neurosci Lett 2003;339:91-4.

79. Biernaskie J, Chernenko G, Corbett D. Efficacy of rehabilitative experience declines with time after focal ischemic brain injury. J Neurosci 2004;24:1245-54.

80. Liu H, Chen L, Zhang G, et al. Scalp Acupuncture Enhances the Functional Connectivity of Visual and Cognitive-Motor Function Network of Patients with Acute Ischemic Stroke. Evid Based Complement Alternat Med 2020;2020:8836794.

81. Wu Q, Chen J, Yue J, et al. Electroacupuncture improves neuronal plasticity through the A2AR/cAMP/ PKA signaling pathway in SNL rats. Neurochem Int 2021;145:104983.

82. Belskaya GN, Stepanova SB, Makarova LD, et al. Acupuncture in the prevention and treatment of stroke: a review of foreign studies. Vopr Kurortol Fizioter Lech Fiz Kult 2020;97:68-77.

83. Wu Y, Hu R, Zhong X, et al. Electric Acupuncture Treatment Promotes Angiogenesis in Rats with Middle Cerebral Artery Occlusion Through EphB4/EphrinB2 Mediated Src/PI3K Signal Pathway. J Stroke Cerebrovasc Dis 2021;30:105165.

84. Li D, Chen QX, Zou W, et al. Acupuncture promotes functional recovery after cerebral hemorrhage by upregulating neurotrophic factor expression. Neural Regen Res 2020;15:1510-7. 